\title{
The Effects of Virtual Reality Games in Posture Correction Exercise on the Posture and Balance of Patients with Forward Head Posture
}

\author{
Ho-Hee Son, PT, PhD $\dagger$ \\ Department of Physical Therapy, College of Health Sciences, Catholic University of Pusan
}

Received: January 22, 2020 / Revised: January 31, 2020 / Accepted: February 24, 2020

(c) 2020 J Korean Soc Phys Med

\section{| Abstract |}

PURPOSE: This study examined the effects of posture improvement exercise using virtual reality programs on the posture and balance of patients with forward head postures.

METHODS: Thirty men and women in their $20 \mathrm{~s}$, who had a forward head posture, were divided randomly into a group with posture correction exercise and a group with posture correction exercise combined with virtual reality programs. The posture correction exercise was composed of squats, XCO training, and chin-tuck exercise. In contrast, exercise with virtual reality games involved the Hot Squat, Climbey, and Baskhead programs while wearing a headset. Both groups performed the exercises 15 min a day, three times per week, for four weeks. The balance ability, distance between the acromion and earlobe, and neck joint range of motion were assessed before and after the exercises.

RESULTS: Both groups showed significant reductions in the distance between the acromion and the earlobe, along with

$†$ Corresponding Author : Ho-Hee Son

sonhh@cup.ac.kr, https://orcid.org/0000-0003-0905-6484

This is an Open Access article distributed under the terms of the Creative Commons Attribution Non-Commercial License (http://creativecommons.org/licenses/by-nc/3.0) which permits unrestricted non-commercial use, distribution, and reproduction in any medium, provided the original work is properly cited. significant improvements in the range of joint motion. The group that performed the virtual reality exercises showed a significant increase in the limit of stability. Both groups showed a significant decrease in the sway length. In contrast, the group given the virtual reality exercises showed a significant reduction in the sway speed while standing with their eyes closed.

CONCLUSION: Exercise applying virtual reality programs can be used in clinical and home programs to correct the postures of individuals with a forward head posture because they can trigger interest in inducing active participation.

Key Words: Forward head posture, Balance, Posture, Virtual reality

\section{Introduction}

The forward head posture (FHP) refers to a posture with the head relatively forward in relation to the coronal plane of the human body, and its representative posture is the turtleneck syndrome [1]. In FHP, the center of the body moves forward as the centerline of the head moves forward [2]. In a normal posture, however, the body structures, such as the neck, shoulders, and upper limbs, always maintain constant tension to support the head, which accounts for approximately one-seventh of the body weight. 
In FHP, however, approximately 3.6 times greater pressure is loaded on the neck than that in the correct posture [3]. Therefore, postural deformation and pain in the neck can develop if the neck supports the increased weight of the head for a prolonged period [4].

Many people, who do not exercise and have incorrect postures form the prolonged use of smartphones and computers, experience FHP. Recent studies on FHP have reported that habitual FHP shortened the back head-to-neck muscles, in which the head is extended backward to maintain sight, resulting in a shortening of the extensor and elongation of the flexor of the neck [5,6]. If this posture persists for an extended time, it can lead to the loss of normal forward flexion and muscle weakness [7]. In addition, the persistence of FHP compromises balance because of a muscular imbalance, which may cause a loss of the proprioceptive sense in the joint, which also decreases balance [8].

To improve FHP, various tools, such as posture correction devices, sneakers, and posture corrector belts, as well as other methods, such as posture-correction exercises, Pilates, and core exercises, have recently been utilized. According to Lee [2], Pilates, which involves muscle stretching with the co-activation of the core muscles through a focus on breathing techniques, reduces muscle tension by stabilizing the cervical spine and inducing postural changes and alignments of the body. Kim [9] reported that ballet improves the muscular strength, flexibility, and endurance of individuals with FHP through repetitive movements from the basic spine erecting posture, which further promotes a healthy body shape and helps maintain a correct posture. Therefore, a range of exercises can relieve the symptoms of FHP. Sim et al. [10] conducted proprioceptive balance exercises on balance pads to promote posture and balance in patients with turtleneck syndrome and reported a positive effect on posture correction.

Regarding the treatment methods for people with FHP, asymmetrical muscle strengthening/relaxation, balance, and core exercises are generally prescribed to patients so that they can maintain a correct posture on their own. On the other hand, they are unlikely to continuously perform such posture-correction exercises for a long time if they lose interest or have not developed an exercise habit; thus, people with FHP would show little benefit from the exercises.

Recently, virtual reality systems have been used in assessments and treatments in the field of rehabilitation. According to Kim [11], who applied virtual reality training, this type of training positively affected the balance and gait of stroke patients. Lee et al. [12] also reported improvements in the physical function and quality of life of patients with chronic low back pain. Virtual reality programs reduce the risks that patients face in real life and provide them with the opportunities to experience situations that rarely occur in real life in a safe setting. In addition, virtual reality programs promote a sense of enjoyment and motivation for treatment, which can lead patients to participate voluntarily in the treatments $[13,14]$.

As described earlier, treatments combined with virtual reality programs were recently demonstrated to have therapeutic effects on a range of patients, such as those with stroke or chronic low back pain. On the other hand, no study has applied posture correction exercises combined with virtual reality games to correct FHP.

To improve the posture and balance of people with FHP, the present study performed posture correction exercises on an unstable support surface and examined the effect of posture correction exercises combined with virtual reality programs on FHP.

\section{Methods}

\section{Subjects}

The present study was performed with 30 college students of C University in Busan who volunteered to 
Table 1. Characteristics of Subjects

\begin{tabular}{cccc}
\hline Variables & Group I (n=15) & Group I $(\mathrm{n}=15)$ & $\mathrm{P}(\mathrm{X})$ \\
\hline Sex (Male / Female) & $7 / 8$ & $8 / 7$ & .704 \\
Age (years) & $22.53 \pm 1.25$ & $21.93 \pm 2.54$ & .714 \\
Height (cm) & $168.93 \pm 12.89$ & $166.06 \pm 7.76$ & .243 \\
Weight (kg) & $63.86 \pm 9.42$ & $62.46 \pm 8.44$ & .151 \\
Distance Between the Acromion and the Earlobe $(\mathrm{cm})$ & $3.77 \pm 1.26$ & $4.24 \pm 1.35$ & .101 \\
\hline
\end{tabular}

Group I : Posture correction exercise group

Group $\amalg$ : Posture correction exercise combined with virtual reality programs group

participate in the study. They explained the purpose and procedures of the study before starting the experiments. The inclusion criteria were as follows: age of 20-24 years, distance of $\geq 2 \mathrm{~cm}$ between the acromion and the earlobe when they were positioned perpendicular to the ground [15], and a visual analog scale (VAS) score for shoulder pain of at least five points. On the other hand, those who had a history of surgery within the last six months, issues in the vestibular organ, presence of dizziness, or cybersickness during virtual reality games, were excluded. All participants gave their written informed consent to participate in the study.

Depending on the intervention method, the 30 subjects were divided randomly into the posture-correction exercise group (group I) and the "posture correction exercise combined with virtual reality games" group (group II). Table 1 lists the subjects' demographic information.

\section{Contents and Procedures}

The subjects were allocated randomly to a group with posture correction exercises without virtual reality programs (15 subjects) and a group with posture correction exercises combined with virtual reality programs (15 subjects). The treatments were performed for $15 \mathrm{~min}$ per session, three times a week for four weeks. The posture correction exercise program was composed of chin tucks for five minutes, XCO exercise for five minutes, and squats for five minutes. In contrast, the posture correction exercise program combined with virtual reality games was composed of Baskhead, Climbey, and Hot Squat game training for five minutes each (Fig. 1).

To analyze posture, the distance between the acromion and earlobe before and after exercise was measured in both groups. To assess the neck joint range of motion, the neck flexion and extension, and lateral (left, right) bending angles were measured. Finally, the balance was evaluated using Biorescue, which is a device for measuring the static and dynamic balances.

\section{1) Intervention Methods}

(1) Posture Correction Exercise

Posture correction exercise was performed on the balance pads for proprioceptive sensory training, which included three exercises, as shown in Fig. 1.

\section{(1) Squats}

The subjects stood with their feet shoulder-width apart on balance pads. They lowered their hips from a standing position until their thighs were lowered to the height of the knees and then stood back up. Each of these was performed for four seconds each time for five minutes.

\section{(2) XCO Training}

While holding styrofoam dumbbells in their hands, the subjects stood with their feet shoulder-width apart on balance pads. They performed one shoulder flexion within 
Posture correction exercise

a. Squats $(5 \mathrm{~min})$

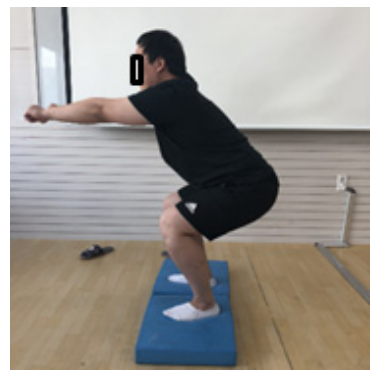

b. X-CO $(5 \mathrm{~min})$

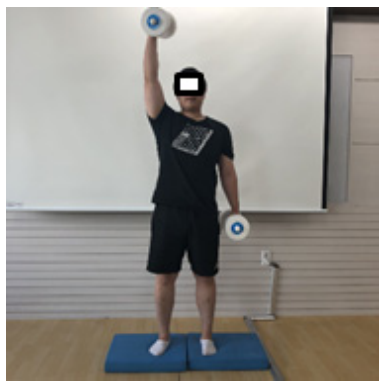

c. Chin tuck exercises $(5 \mathrm{~min})$

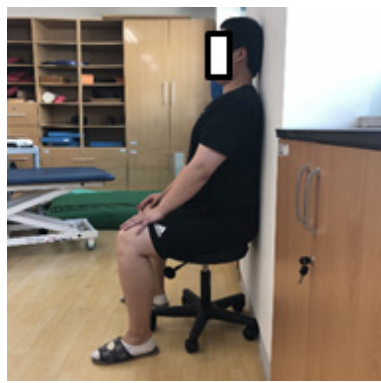

Posture correction exercise combined with virtual reality programs

a. Hot Squat $(5 \mathrm{~min})$

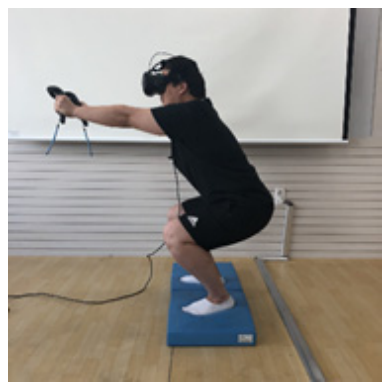

b. Climbey (5 $\mathrm{min})$

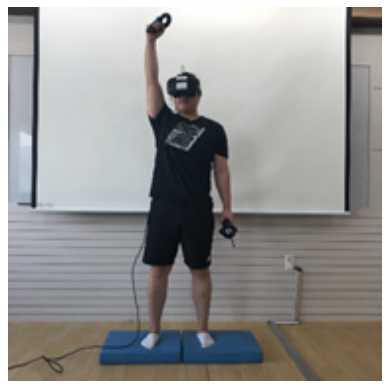

c. Baskhead (5 min)

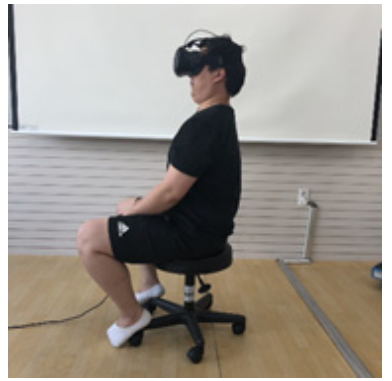

Fig. 1. Intervention program.

two seconds, alternating between the left and right shoulders for five minutes.

\section{(3) Chin Tucks}

With their heels placed against the wall, the subjects tucked their chin in an upright standing position to keep their head touching the wall for five minutes.

(2) Posture Correction Exercise using Virtual Reality Games Posture correction exercises in conjunction with virtual reality games using a head-mounted display (VIVE PORT, Taiwan) were also performed on the balance pads. For these, software programs similar to the motions in the postures of the exercise program in the correction exercise group were selected (Fig. 2).

\section{(1) Hot Squat Exercise}

While wearing a virtual reality headset in the standing position on balance pads, the subjects performed squats while pushing the device to pass through the tunnels that 


\begin{tabular}{|c|c|c|}
\hline Hot squat & Backhead & Climbey \\
\hline & & \\
\hline
\end{tabular}

Fig. 2. Virtual reality game screenshot.

appeared on the screen of the device. Once the subjects failed by hitting the wall, they repeated the game for five minutes.

\section{(2) Climbey Exercise}

While wearing a virtual reality headset in a standing position on balance pads, the subjects performed rock climbing by holding the device. Upon completion, they repeated the same game from the beginning for five minutes.

\section{(3) Baskhead Exercise}

While wearing a virtual reality headset in the sitting position on a chair, the subjects caught basketballs thrown toward the rim by moving only their head for 45 seconds each time for five minutes.

\section{Measurement Methods}

All evaluations were performed three times, and the mean values were recorded.

\section{1) Posture Analysis}

In the upright standing position, the perpendicular distance from the ruler to the ground at the acromion and earlobe was measured. The person was considered as having FHP if the distance was $\geq 2 \mathrm{~cm}$.

\section{2) Measurements of the Neck Joint Range of Motion}

The best range of motion, which can be developed using the muscular strength and willpower in an upright sitting position, was measured. This experiment measured the neck flexion and extension angles, and the lateral, left, and right bending angles.

\section{3) Evaluation of Dynamic Balance}

During the measurements, the subjects were instructed to keep their eyes forward in the upright standing position with their feet apart at an angle of approximately $30^{\circ}$. The measurement method was explained on the monitor, and an example was shown, followed by actual measurements.

The subjects were asked to maintain their balance using the ankle strategy while moving toward the direction shown on the monitor in the upright standing position. The limit of stability in the upright position that can move the center of mass to the maximum range was measured by centimeter, and then the balance was compared.

\section{4) Evaluation of Static Balance}

The subjects faced forward in the upright standing position for one minute each with their eyes either open or closed; during this time, the total path length of the body's center of mass (CoM) was measured.

\section{Experimental Tools}

\section{1) Head Mount Display}

A head-mount display, which was composed of a headset, controller, and base station, was used to connect the virtual reality programs to the exercise program. The subjects in group II performed exercises with the movement 
range, and the floor and ceiling heights were set in accordance with their body configurations.

\section{2) Biorescue}

Biorescue (AP1153 Biorescue, France) was used to measure the dynamic and static balances of the subjects before and after the exercises. The limit of stability was used to assess the dynamic balance, while a sway test was used to evaluate the static balance. The measurements were taken with the heels spaced $3 \mathrm{~cm}$ apart, and both feet angled $30^{\circ}$ away from the centerline.

\section{3) Balance Pads}

Balance pads (Airex, Switzerland) were used to provide unstable support surfaces. Each subject used two balance pads and placed one leg on each pad for the exercises.

\section{4) Rulers}

In the upright standing position, the distance between the perpendicular line of the ruler at the acromion and that at the earlobe was measured.

\section{5) Goniometer}

A goniometer was used to measure the neck joint range of motion. For neck joint flexion and extension, the axis was set on the ear hole. The fixed arm was placed perpendicular to the ground. The moving arm was placed on the nose tip of the subject. For lateral bending of the neck joint, the axis was set on the seventh cervical spine. The fixed arm was placed perpendicular to the ground with the spinous process on the spine. The moving arm was placed at the top of the head. For the left and right bending of the neck joint, the axis was set on the top of the head.
The fixed arm was placed in parallel to the line connecting both acromia. The moving arm was set on the nose tip of the subject for the measurements during movements.

\section{Data Analysis}

All data from the present study were subjected to Windows SPSS/PC 21.0, a statistical program, through which the descriptive statistic values (mean $\pm \mathrm{SD}$ ) were calculated. A paired t-test was performed to examine the differences depending on the intervention method (posture correction exercise vs. posture correction exercises combined with virtual reality games). An independent t-test was performed to compare the differences between the two groups. A p-value $<.05$ was considered significant.

\section{Results}

1. The pre- and post-intervention outcomes of the neck joint range of motion in the group with posture correction exercise (group I) and the group with posture correction exercises combined with virtual reality games (group II)

With respect to the distance between the acromion and earlobe, groups I and II showed significant differences in the neck joint range of motion before and after the experiments $(\mathrm{p}<.05)($ Table 2$)$.

2. Pre- and Post-intervention Outcomes of the Posture Analysis in Groups I and II

Regarding the neck joint range of motion, groups I and II showed significant differences in the neck joint range of motion between before and after the experiments ( $p$ $<.05$ ) (Table 3).

Table 2. Comparison of the Changes in Distance Between the Acromion and the Earlobe in Each Group

\begin{tabular}{lcccccc}
\hline Variables & Group & Pre & Post & Post-pre & $\mathrm{t}$ & $\mathrm{p}$ \\
\hline \multirow{2}{*}{ Distance } & Group I & $3.77 \pm 1.26$ & $1.47 \pm .47$ & -2.32 & 9.971 & $.000^{*}$ \\
& Group II & $4.24 \pm 1.35$ & $1.54 \pm 1.24$ & -2.73 & 7.270 & $.000^{*}$ \\
\hline
\end{tabular}

$($ mean $\pm \mathrm{SD}, * \mathrm{p}<.05)$ 
Table 3. Comparison of Neck ROM for Each Group

\begin{tabular}{|c|c|c|c|c|c|c|}
\hline \multicolumn{2}{|c|}{ Variables } & Pre & Post & Post-pre & $\mathrm{t}$ & $\mathrm{p}$ \\
\hline \multirow{2}{*}{ Flex. } & Group I & $26.53 \pm 8.75$ & $30.93 \pm 10.09$ & 4.40 & -1.657 & .121 \\
\hline & Group $\amalg$ & $26.06 \pm 14.46$ & $32.33 \pm 13.47$ & 6.66 & -1.942 & .073 \\
\hline \multirow{2}{*}{ Ext. } & Group I & $29.73 \pm 10.26$ & $36.40 \pm 11.55$ & 6.67 & -2.304 & $.037^{*}$ \\
\hline & Group $\amalg$ & $30.53 \pm 16.07$ & $39.66 \pm 10.08$ & 9.13 & -2.246 & $.042 *$ \\
\hline \multirow{2}{*}{ Lt. bend. } & Group I & $32.13 \pm 7.89$ & $29.93 \pm 7.61$ & -2.20 & .838 & .416 \\
\hline & Group $\Pi$ & $29.66 \pm 7.89$ & $32.26 \pm 5.48$ & 2.60 & -1.481 & .159 \\
\hline \multirow{2}{*}{ Rt. bend. } & Group I & $30.80 \pm 7.22$ & $33.20 \pm 9.48$ & 2.40 & -.997 & .336 \\
\hline & Group $\amalg$ & $31.86 \pm 8.73$ & $32.40 \pm 8.79$ & .53 & -.255 & .802 \\
\hline \multirow{2}{*}{ Lt. rot. } & Group I & $50.01 \pm 17.42$ & $49.60 \pm 10.71$ & -.40 & .093 & .928 \\
\hline & Group $\amalg$ & $52.66 \pm 13.61$ & $51.66 \pm 11.63$ & -1.00 & .319 & .754 \\
\hline \multirow{2}{*}{ Rt. rot. } & Group I & $53.80 \pm 18.73$ & $56.03 \pm 13.61$ & 2.20 & -.374 & .714 \\
\hline & Group $\amalg$ & $55.20 \pm 15.82$ & $52.53 \pm 10.20$ & -2.66 & .696 & .498 \\
\hline
\end{tabular}

$($ mean $\pm \mathrm{SD}, * \mathrm{p}<.05)$

Table 4. Comparison of the Limit of Stability for Each Group

$(\mathrm{cm})$

\begin{tabular}{|c|c|c|c|c|c|c|}
\hline \multicolumn{2}{|c|}{ variables } & Pre & Post & Post-pre & $\mathrm{t}$ & $\mathrm{p}$ \\
\hline \multirow{2}{*}{ Lt. } & Group I & $5744.80 \pm 1403.46$ & $5610.93 \pm 2027.22$ & -.133 .86 & .327 & .749 \\
\hline & Group $\Pi$ & $5130.80 \pm 1511.90$ & $6379.93 \pm 1793.15$ & 1249.13 & -2.49 & $.026^{*}$ \\
\hline \multirow{2}{*}{ Rt. } & Group I & $6106.86 \pm 2121.25$ & $6460.73 \pm 2206.69$ & 353.86 & -.471 & .645 \\
\hline & Group $\mathbb{I}$ & $6250.70 \pm 2570.35$ & $5923.26 \pm 1485.57$ & -327.43 & .709 & .490 \\
\hline \multirow{2}{*}{ Forward } & Group I & $7403.06 \pm 2056.76$ & $7048.33 \pm 2292.74$ & -354.73 & .715 & .486 \\
\hline & Group $\Pi$ & $6731.33 \pm 1984.21$ & $7337.13 \pm 2058.93$ & 605.80 & -1.04 & .315 \\
\hline \multirow{2}{*}{ Backward } & Group I & $4581.93 \pm 1697.82$ & $5045.73 \pm 2417.43$ & 463.80 & -.933 & .366 \\
\hline & Group $\amalg$ & $4459.93 \pm 2282.86$ & $4950.13 \pm 2127.83$ & 490.20 & -1.00 & .332 \\
\hline \multirow{2}{*}{ Total } & Group I & $11985.03 \pm 2725.94$ & $12149.13 \pm 3872.29$ & 164.13 & -.184 & .857 \\
\hline & Group $\square$ & $10359.56 \pm 4432.48$ & $12329.06 \pm 3127.57$ & 1969.50 & -2.60 & $.021 *$ \\
\hline
\end{tabular}

$($ mean $\pm \mathrm{SD}, * \mathrm{p}<.05)$

3. Pre- and Post-intervention Outcomes in the Limit of Stability Test in Groups I and II

Group I showed no significant difference in the statistical analysis of the limit of stability between before and after the experiments.

Statistical analysis of the limit of stability revealed group II to have significant differences in the limit of stability on the left side and in total area $(p<.05)$ (Table 4).

\section{Pre- and Post-intervention Outcomes in the Sway Test in Groups I and II}

In statistical analysis of the balance, group I showed significant differences in the sway length between before and after the experiments, both with their eyes opened and closed, and in the sway speed with their eyes closed ( $p$ $<.05)$.

Group II showed significant differences in the sway length between before and after the experiments with their 
Table 5. Comparison of Sway Test for Each Group

\begin{tabular}{|c|c|c|c|c|c|c|c|}
\hline & Variables & & Pre & Post & Post-pre & $\mathrm{t}$ & $\mathrm{p}$ \\
\hline \multirow{4}{*}{ Sway Area } & \multirow{2}{*}{ Eyes Open } & Group I & $88.53 \pm 72.47$ & $105.66 \pm 178.49$ & 17.13 & -.388 & .704 \\
\hline & & Group $\amalg$ & $214.26 \pm 302.42$ & $182.40 \pm 210.04$ & -31.86 & .583 & .569 \\
\hline & \multirow{2}{*}{ Eyes Closed } & Group I & $90.46 \pm 76.21$ & $88.66 \pm 92.88$ & -1.83 & .073 & .943 \\
\hline & & Group $\amalg$ & $165.93 \pm 282.35$ & $86.46 \pm 105.05$ & -79.46 & 1.10 & .289 \\
\hline \multirow{4}{*}{ Sway Length } & \multirow{2}{*}{ Eyes Open } & Group I & $32.11 \pm 14.20$ & $21.75 \pm 8.50$ & -10.36 & 2.732 & $.016^{*}$ \\
\hline & & Group $\amalg$ & $32.60 \pm 11.69$ & $24.66 \pm 7.06$ & -7.94 & 3.438 & $.004 *$ \\
\hline & \multirow{2}{*}{ Eyes Closed } & Group I & $27.60 \pm 6.77$ & $20.66 \pm 6.58$ & -6.94 & 3.473 & $.004^{*}$ \\
\hline & & Group $\Pi$ & $28.14 \pm 11.21$ & $22.05 \pm 4.58$ & -6.08 & 2.283 & $.039 *$ \\
\hline \multirow{4}{*}{ Sway Speed } & \multirow{2}{*}{ Eyes Open } & Group I & $.52 \pm .23$ & $.42 \pm .28$ & .10 & 1.195 & .251 \\
\hline & & Group $\amalg$ & $.54 \pm .19$ & $.42 \pm .10$ & -.12 & 3.056 & $.009^{*}$ \\
\hline & \multirow{2}{*}{ Eyes Closed } & Group I & $.46 \pm .10$ & $.34 \pm .11$ & -.11 & 3.692 & $.002 *$ \\
\hline & & Group $\amalg$ & $.48 \pm .18$ & $.36 \pm .09$ & .12 & 2.553 & $.023 *$ \\
\hline
\end{tabular}

$($ mean $\pm \mathrm{SD}, * \mathrm{p}<.05)$

eyes opened and closed $(\mathrm{p}<.05)$. Significant differences in sway speed were also observed with both eyes opened and closed $(\mathrm{p}<.05)($ Table 5).

\section{Discussion}

This study examined the effects of posture correction exercises combined with virtual reality games on FHP. Thirty subjects in their twenties who had turtleneck syndrome performed the posture correction exercises, either with or without virtual reality games, during which the neck joint range of motion and limit of stability were measured. A sway test and posture analysis (measurement of the distance between the acromion and the earlobe) were performed.

As a result, group I showed an increase in the neck joint range of motion, a decrease in the distance between the acromion and the earlobe, a significant decrease in the sway length both with the eyes opened and closed, and a significant decrease in sway speed with the eyes closed. Group II showed an increase in the neck joint range of joint motion, a decrease in distance between the acromion and the earlobe, a significant reduction in sway length in the sway test both with eyes opened or closed, and a significant decrease in the sway speed in all states.

Balance exercise, which is one of the posture correction exercises often prescribed to people with FHP, has been used frequently because it is simple, safe, and affordable. Sim et al. [10] examined the effect of proprioceptive balance exercise on turtleneck syndrome, targeting 20 information technology employees. They reported improvements in the height balance between the shoulder and pelvis after the exercise, as well as significant positive changes in the angle and distance of the neck with turtleneck syndrome. In addition, to improve the physical balance and turtleneck syndrome, Han and Song [16] asked college students to perform combined exercises, consisting of four steps with the main exercise, including squat, leg press, bench press, and shoulder press, as well as warm-up, aerobic, and cool-down exercises. The combined exercises improved the physical balance and reduced the forward flexion of the neck joint, and had positive effects on posture correction. In the present study, group I showed a significant reduction (by $-2.30 \mathrm{~cm}$ on average) in the distance between the acromion and earlobe after the exercise. Group II also showed a statistically significant decrease in distance, by $-2.70 \mathrm{~cm}$ 
on average, showing its positive effect on FHP correction, which supports the results of preceding studies [16].

In residents around a university who were diagnosed with turtleneck syndrome, Young Hwan Kim et al. [17] examined the effects of a chiropractic and theratainment exercise program on the balancing ability in turtleneck syndrome and reported significant positive effects not only on neck bone misalignment correction but also on the dynamic and static balances. They reported a number of similarities with the exercise program, including chin-tuck exercise and exercise with both hands stretched forward. Although not significant in the present study, groups I and II generally showed improvement in the maximum stability in dynamic balance. Group II also showed significant differences in maximum stability on the left side and total area, which supports the claims of previous studies [17].

In the sway test performed in the present study, groups I and II showed improvements in balance. Group I had significant values in three of the six measured variables, whereas group II showed significant outcomes for four variables. The positive effects of the virtual reality games during the exercise were attributed to the following reasons. Once subjects were given a goal, Thomas et al. [18] reported that the ankles, knees, hip, spine, and shoulders had greater movements when performed through virtual reality games than when performed in real-life situations. In addition, Kishore et al. [19] reported that exercises combined with virtual reality games motivated participation in physical activities, and were effective in improving exercise compliance and outcomes. Similarly, in the present study, the visual setting fostered by the virtual reality games appeared to affect the subjects' performance of the tasks. Moreover, the greater movements induced by the virtual reality games, not by the simple posture correction exercise program, should have positive effects on the balance of people with FHP. Moreover, the virtual reality games require more attention than the simple exercises because of the sight blocking by wearing the headset and space sense in virtual reality. Therefore, four weeks of intervention appeared to elicit a further positive change in balance.

In measurements of the neck joint range of motion, both groups showed similar ranges of motion in the five items except for the neck joint range of motion. In addition, all the subjects in the present study had a normal range of joint motion. Therefore, it is difficult to prove the significance of the differences between the pre- and post-intervention outcomes in terms of the range of motion.

The present study had some limitations. The results are difficult to generalize owing to the small number of subjects. As the improvement of FHP was analyzed based only on the differences in balance and distance between the acromion and the earlobe, a further in-depth analysis will be needed. On the other hand, the distance between the acromion and the earlobe is an important tool for diagnosing turtleneck syndrome, and the stress caused by the load on the neck was reduced by the shortened distance. In addition, the games were difficult to choose because of the insufficient number of virtual reality games for medical purposes. The virtual reality games that were most similar to posture correction exercises were chosen, which enabled control of the amount of exercise between the groups for comparative analysis.

Virtual reality-based clinical interventions are expected to become more popular as they become more accessible and affordable, which would increase their potential applications in clinical and home settings [20]. Further research will be needed to develop and generalize more enjoyable and better outcomes through interventions based on gaming concepts using virtual reality beyond conventional posture correction exercises, such as correction, strengthening, and core strengthening exercises.

\section{Conclusion}

The present study was conducted on people with FHP to compare the degree of posture correction by posture 
correction exercises with or without the use of virtual reality programs. Both groups showed significant decreases in the distance between the acromion and the earlobe in the posture analysis. In the balance test, group II showed significant improvement in the left maximum stability and total area measurement. In addition, the posture and balance were improved significantly in the group with the virtual reality games compared to the group without virtual reality games. Therefore, it is expected that exercise programs combined with virtual reality games to correct the posture of people with FHP would trigger interest, which would, in turn, induce active participation in posture-corrective exercise programs, resulting in positive outcomes.

\section{Acknowledgements}

This work was supported by the National Research Foundation of Korea (NRF) funded by the Korea government (MSIT) No. 2017R1C1B5074040.

\section{References}

[1] Griegel-Morris P, Larson K, Mueller-Klaus K, et al. Incidence of common postural abnormalities in the cervical, shoulder, and thoracic regions and their association with pain in two age groups of healthy subjects. Phys Ther. 1992;72(6):425-31.

[2] Lee HJ. Effects of Pilates-Based Exercise on Neck and Shoulder Muscle Architecture, Cervical Alignment and Body Function in Patients with Turtleneck Syndrome. Master's Degree. Sahmyook University. 2018.

[3] Mahmoud NF, Hassan KA, Abdelmajeed SF, et al. The Relationship Between Forward Head Posture and Neck Pain: a Systematic Review and Meta-Analysis. Curr Rev Musculoskelet Med. 2019;12(4):562-77.

[4] Harrison DE, Harrison DD, Betz JJ, et al. Increasing the cervical lordosis with chiropractic biophysics seated combined extension-compression and transverse load cervical traction with cervical manipulation: nonrandomized clinical control trial. J Manipulative Physiol Ther. 2003;26(3):139-51.

[5] Kraus SL. Temporomandibular disorders (2nd ed). New York. Churuchill Livingstone. 1994. pp.xvi, 486 p.

[6] Kocur P, Wilski M, Goliwas M, et al. Influence of Forward Head Posture on Myotonometric Measurements of Superficial Neck Muscle Tone, Elasticity, and Stiffness in Asymptomatic Individuals With Sedentary Jobs. J Manipulative Physiol Ther. 2019;42(3):195-202.

[7] Jull G. Cervical flexor muscle retraining: physiological mechanisms of efficacy. Kinetic Control and Manipulation Association of Chartered Physiotherapists (UK). 2005: L01.

[8] Zarins B, Rowe CR. Current concepts in the diagnosis and treatment of shoulder instability in athletes. Med Sci Sports Exerc. 1984;16(5):444-8.

[9] Kim Y. The effect of ballet program on turtle neck syndrome in office workers. Master's Degree. Hanyang University. 2012.

[10] Sim HP, Yoon HI, Youn IN. The Effect of Vision and Proprioception on Lumbar Movement Accuracy Journal of Korean academy of orthopaedic manual therapy. 2007;13(2):31-44.

[11] Kim JH. Effects of Virtual Reality Program on Standing Balance in Chronic Stroke Patients The journal of Korean society of physical therapy. 2005;17(3):351-67.

[12] Lee SH, KO DS, Jung DI. The Effect of Virtual Reality Experimental Training Using Nintendo Wii Sports Programs on Balance Proficiency of Clients With Chronic Low Back Pain. The Journal of Korean Society of Assistive Technology 2010;2(2):23-9.

[13] Bryanton C, Bosse J, Brien M, et al. Feasibility, motivation, and selective motor control: virtual reality compared to conventional home exercise in children with cerebral palsy. Cyberpsychol Behav. 2006;9(2):123-8.

[14] Sveistrup H. Motor rehabilitation using virtual reality. J Neuroeng Rehabil. 2004;1(1):10. 
[15] Kim J, Kim S, Shim J, et al. Effects of McKenzie exercise, Kinesio taping, and myofascial release on the forward head posture. J Phys Ther Sci. 2018;30(8):1103-7.

[16] Han GS, Song IW. The Effect of Combined Exercise Program on Balance, Shoulder Gradient and Forward Head Posture in College Students Korean Journal of Sports Science. 2017;26(2):1127-37.

[17] Kim YH, Park IY, Khil JH. Effect of Chiropractic and Theratainment Exercise Program on the Balance for the Subjects with Turtle Neck Syndrome. Journal of the Korea Entertainment Industry Association. 2017;11(7):323-33.
[18] Thomas JS, France CR, Leitkam ST, et al. Effects of Real-World Versus Virtual Environments on Joint Excursions in Full-Body Reaching Tasks. IEEE J Transl Eng Health Med. 2016;4:2100608.

[19] Kishore TA, Beddingfield R, Holden T, et al. Task deconstruction facilitates acquisition of transurethral resection of prostate skills on a virtual reality trainer. J Endourol. 2009;23(4):665-8.

[20] Weiss PL, Rand D, Katz N, et al. Video capture virtual reality as a flexible and effective rehabilitation tool. J Neuroeng Rehabil. 2004;1(1):12. 